\title{
Aspectos microbiológicos e imunológicos relacionados à colonização e infecção de pacientes por Acinetobacter baumannii resistente em ambiente nosocomial
}

Microbiological and immunological aspects related to the colonization and infection of patients by resistant Acinetobacter baumannii in nosocomial environment Aspectos microbiológicos e inmunológicos relacionados con la colonización e infección de pacientes por Acinetobacter baumannii resistente en ambiente nosocomial

Cynthia Karolina Rodrigues do Nascimento ORCID: https://orcid.org/0000-0002-2312-2364 Universidade Federal do Delta do Parnaíba, Brasil E-mail: biomedcynthia1@gmail.com

Melissa Macêdo Santos ORCID: https://orcid.org/0000-0003-0774-5577 Secretaria de Saúde de São Raimundo Nonato, Brasil E-mail: mmacedoesantos@gmail.com

Patrícia Maria Costa Oliveira ORCID: https://orcid.org/0000-0001-9910-4552 Universidade Federal do Delta do Parnaíba, Brasil E-mail: tricioliveira@gmail.com

Marcos Vinicius Alves Hiroshi Iomori ORCID: https://orcid.org/0000-0002-9081-1194 Universidade Estácio de Sá, Brasil E-mail: hiroshi.iomori.01@gmail.com

Raylane Meneses Marques dos Santos ORCID: https://orcid.org/0000-0002-9394-9527 Centro Universitário UNINOVAFAPI, Brasil E-mail: raylane.mms@hotmail.com Victor Brito Dantas Martins ORCID: https://orcid.org/0000-0002-6932-915X

Secretaria de Saúde de Pimenteiras, Brasil E-mail: victorbritodantas@gmail.com

Beatriz Ferreira Melo ORCID: https://orcid.org/0000-0003-2551-2398 Laboratório LABCLIN, Brasil E-mail: beatrizmelofe@gmail.com

Antonia Luzia Lima do Nascimento ORCID: https://orcid.org/0000-0001-9740-5669 Universidade Federal do Rio Grande do Sul, Brasil E-mail: antonialuzialimaa@gmail.com

Iane Suellen Pereira dos Santos ORCID: https://orcid.org/0000-0001-6738-1846 Centro Universitário Maurício de Nassau, Brasil E-mail: ianesuellen8@gmail.com

Tacyana de Carvalho Dias ORCID: https://orcid.org/0000-0003-3824-0651 Universidade Federal do Piauí, Brasil E-mail: tacycarvalho@ufpi.edu.br

Messias Henrique da Silva Santos ORCID: https://orcid.org/0000-0002-7454-9157 Universidade Federal do Piauí, Brasil E-mail: messiashenrique2@gmail.com Ayane Araújo Rodrigues ORCID: https://orcid.org/0000-0001-7792-6993 Universidade Federal do Delta do Parnaíba, Brasil

E-mail: ayanerodrigues2012@hotmail.com

Mayara Stefanni de Lacerda Bezerra ORCID: https://orcid.org/0000-0003-0038-3673 Universidade Federal do Delta do Parnaíba, Brasil E-mail: mayarastefanni@ hotmail.com 


\author{
Raíssa Silva Bacelar Andrade \\ ORCID: https://orcid.org/0000-0002-9126-3476 \\ Universidade Federal do Delta do Parnaíba, Brasil \\ E-mail: raissa_sba@hotmail.com \\ Samuel Cabral dos Santos \\ ORCID: https://orcid.org/0000-0002-6250-4912 \\ Universidade Estadual do Piauí, Brasil \\ E-mail: samclsbio@outlook.com
}

\begin{abstract}
Resumo
Introdução: Conforme avançamos no desenvolvimento de antimicrobianos, observamos novas formas de resistência bacteriana decorrente da seletividade proveniente do uso irracional destes fármacos. Emerge uma espécie capaz de causar graves infecções especialmente em ambiente nosocomial: Acinetobacter baumannii. Ao produzir mecanismos de resistência, adapta-se a meios menos favoráveis, o que o torna pauta de debates mundiais no que tange ao futuro da antibioticoterapia por ser responsável por altas taxas anuais de mortalidade decorrente de infecções hospitalares. Apesar disso, há escassez de dados epidemiológicos que promovam a conscientização, especialmente no Brasil. Objetivo: Reunir informações acerca dos aspectos imunológicos decorrentes da colonização por esta bactéria e mecanismos de defesa envolvidos nos casos de indivíduos infectados. Metodologia: Revisão de literatura através da análise de artigos dos bancos Pubmed e Scielo, obteve-se informações sobre formas de transmissão, doenças associadas, reações imunológicas causadas e principais formas de resistência. Resultados: Frequentemente, as Unidades de Terapia Intensiva (UTIs) brasileiras detectam casos de Infecções Relacionadas à Assistência à Saúde (IRAS). Nos casos em que as cepas de Acinetobacter baumannii são responsáveis, geralmente apresentam resistência aos antimicrobianos disponíveis, causando altos custos financeiros no tratamento e taxa de mortalidade de aproximadamente $47 \%$ por conta da fragilidade imunológica e realização de procedimentos invasivos. Conclusão: O paciente colonizado por A. baumannii se torna um agente de transmissão indireta de patógeno ao ambiente hospitalar e as infecções são severas, portanto, elucidar os modos de transmissão e perfil de resistência é de extrema relevância clínica e epidemiológica, considerando a escassez de dados epidemiológicos.
\end{abstract}

Palavras-chave: Acinetobacter baumannii; Resistência bacteriana; Imunologia; Antibioticoterapia.

\begin{abstract}
Introduction: As we progress in the development of antimicrobials, we observe new forms of bacterial resistance due to the selectivity resulting from the irrational use of these drugs. A species emerges capable of causing serious infections, especially in a nosocomial environment: Acinetobacter baumannii. By producing resistance mechanisms, it adapts to less favorable means, which makes it the subject of worldwide debates regarding the future of antibiotic therapy as it is responsible for high annual mortality rates due to hospital infections. Despite this, there is a shortage of epidemiological data that promote awareness, especially in Brazil. Objective: To gather information about the immunological aspects resulting from colonization by this bacterium and defense mechanisms involved in cases of infected individuals. Methodology: Literature review through analysis of articles from Pubmed and Scielo banks, information was obtained on forms of transmission, associated diseases, immunological reactions caused and main forms of resistance. Results: Brazilian Intensive Care Units (ICUs) frequently detect cases of Health Care Related Infections (IRAS). In cases where the strains of Acinetobacter baumannii are responsible, they usually show resistance to the available antimicrobials, causing high financial costs in the treatment and a mortality rate of approximately $47 \%$ due to the immunological fragility and carrying out invasive procedures. Conclusion: The patient colonized by $A$. baumannii becomes an agent of indirect transmission of pathogen to the hospital environment and infections are severe, therefore, elucidating the modes of transmission and resistance profile is of extreme clinical and epidemiological relevance, considering the scarcity of epidemiological data.
\end{abstract}

Keywords: Acinetobacter baumannii; Basterial resistance; Immunology; Antibiotic therapy.

\title{
Resumen
}

Introducción: À medida que avanzamos en el desarollo de antimicrobianos, observamos nuevas formas de resisitencia bacteriana debido a la selectividad resultante del uso irracional de estes fármacos. Emerge una especie capaz de causar infecciones graves, especialmente en un ambiente nosocomial: Acinetobacter baumannii. Al producir mecanismos de resistência, se adapta a medios menos favorables, lo que la convierte em objeto de debates mundiales sobre el futuro de la terapia antibiótica, ya que es responsable de altas tasas de mortalidade anual por infecciones hospitalarias. A pesar de esto, hay una escassez de datos epidemiológicos que promuevan la consciência, especialmente en Brasil. Objetivo: Recoger información sobre los aspectos inmunológicos derivados de la colonización por esta bacteria y los mecanismos de defensa involucrados en los casos de individuos infectados. Metodología: Revisión de la literatura mediante el análisis de artículos de los bancos Pubmed y Scielo, se obtuvo información sobre formar de transmisión, enfermedades asociadas, reacciones inmunológicas provocadas y principales formas de transmisión, enfermidades associadas, reacciones inmunológicas provocadas y principales formas de resistencia. Resultados: Las Unidades de Cuidados Intensivos (UCI) brasileñas detectan con frecuencia casos de Infecciones Relacionadas con la Atención de la Salud (IRAS). En los casos que las cepas de Acinetobacter 
baumannii son las responsables, suelen mostrar resistencia a los antimicroboanos disponibles, provocando altos costes económicos en el tratamiento y una tasa de mortalidad de aproximadamente el $47 \%$ por la fragilidad inmunológica y la realización de procedimientos invasivos. Conclusión: El paciente colonizado por A. baumannii se convierte en un agente de transmisión indirecta de patógeno al ambiente hospitalario y las infecciones son graves, por lo que dilucidar los modos de transmisión y el perfil de resistencia es de extrema relevancia clínica y epidemiológica, considerando la escasez de datos epidemiológicos.

Palabras clave: Acinetobacter baumannii; Resistencia bacteriana; Inmunología; Terapia con antibióticos.

\section{Introdução}

No século XXI, concomitante ao avanço científico e tecnológico construído no ramo da microbiologia, observamos também o surgimento de uma grande problemática no que diz respeito à manutenção da saúde de pacientes fragilizados: a resistência bacteriana decorrente da maior pressão seletiva proveniente do uso de antibióticos. À medida que novas infecções são observadas e documentadas, novos microrganismos ou formas de resistência têm sido descobertos. Desta forma, as infecções têm ressurgido com novos desafios à saúde pública, principalmente quando se trata de pacientes que necessitam de terapia intensiva (European Center For Disease Prevention and Control, 2020).

Diante disto, as infecções oportunistas são consideradas um dos mais significativos riscos aos pacientes hospitalizados quando observamos as taxas de mortalidade. A realidade fica ainda mais desafiadora quando os patógenos responsáveis, através da variabilidade genética e capacidade de mutação mais rápida que o desenvolvimento de novas formas de tratamento, conseguem se sobrepor aos métodos terapêuticos de novas formas de tratamento, mais empíricos (Scarcella, et al, 2017).

Um dos gêneros mais emergentes na condição de patógenos nosocomiais resistentes é o Acinetobacter. Compreendendo atualmente 31 espécies de bactérias, tal grupo foi primeiramente descrito como patógenos hospitalares oportunistas, desde que, por volta de 1970, numerosos surtos hospitalares foram documentados e atribuídos principalmente à espécie Acinetobacter baumannii. Esta pode colonizar a nasofaringe, cavidade oral, e tratos gastrointestinal, respiratório e vaginal, causando, eventualmente, pneumonias, complicações pós-cirúrgicas, danos teciduais na pele, ossos, sistema nervoso central e em casos mais graves, bacteremia. Geralmente, a colonização de pacientes imunossuprimidos e fragilizados precedem infecções (Dessel, et al., 2004; Manual De Orientação Para Controle da Disseminação de Acinetobacter, 2007).

É considerado um patógeno bacteriano emergente por apresentar elevada patogenicidade e alta capacidade de disseminação entre pacientes hospitalizados e acometidos de enfermidades mais severas, além da colonização dos profissionais de saúde, o que pode gerar surtos e epidemias (Wroblewska, et al., 2004). No Brasil, um estudo realizado em Uberaba-MG, mostrou que 60,5\% de Acinetobacter spp. isoladas de pacientes internados em um hospital universitário eram resistentes a carbapenems, drogas que representam a primeira linha de combate mais utilizado. Por ser uma taxa significativa, este resultado demanda que medidas de prevenção e controle das infecções nosocomiais sejam implantadas de forma efetiva, assim como as estratégias de tratamento com antibióticos (Ciello \& Araújo, 2016).

Acinetobacter baumannii tem forma de bacilo em sua fase de crescimento e cocóide na fase estacionária, gramnegativo, oxidase-negativo, imóvel, é a segunda espécie não fermentadora mais isolada em seres humanos por estar presente na microbiota da pele em indivíduos não hospitalizados (Perez, et al., 2007). As espécies de origem humana apresentam um bom crescimento em meios sólidos já frequentemente utilizados em laboratórios de microbiologia clínica: Ágar Sangue e MacConkey a $37^{\circ} \mathrm{C}$ (Oplustil, 2004).

No meio ambiente, encontra-se este microrganismo no solo fazendo decomposição de compostos aromáticos, na água, e em animais, sendo caracterizado como um microrganismo ubíquo (Merkier \& Centrón, 2006). Por serem, eventualmente, componentes da microbiota da pele humana, mesmo em pacientes saudáveis, são frequentemente isolados em outros sítios, porém, quando em trato respiratório de pacientes em ambiente hospitalar, são considerados patógenos oportunistas. Em decorrência da fragilização da primeira barreira antimicrobiana, a epiderme, somada à supressão imunológica enfrentada por 
pacientes internados, sugere-se que a colonização da pele pode ser considerada fator de risco para o desenvolvimento de infecções graves, como pneumonias e bacteremias. Além da facilidade em infectar seres humanos, este patógeno é facilmente encontrado, também, em equipamentos hospitalares formando biofilme (Fournier \& Richet, 2006).

A respeito de sua nutrição, o ferro é um importante fator para o crescimento e sobrevivência desta bactéria no ambiente e no hospedeiro, servindo de fonte de energia, contribuindo com a expressão de sua virulência. Sua absorção é realizada através de sideróforos, acinetobactinas e proteínas modificadoras de receptores de ferro presentes na membrana externa. Suas poucas exigências nutricionais somadas aos fatores de resistência a antimicrobianos como produção de betalactamases cromossomais ou plasmidiais e, hiper expressão do gene cefalosporinase cromossomal do tipo C ( $A m p \mathrm{C})$, alteração de porinas e bomba de efluxo, combinadas com tolerância à diversas condições físicas como umidade, temperatura e pH fazem da A. baumannii um patógeno emergente de grande importância clínica (Goel \& Kapil, 2001).

\section{Metodologia}

Trabalho construído a partir de uma revisão de literatura através de artigos publicados nas bases de dados Pubmed e Scielo e dados epidemiológicos oficiais. A princípio, foram consideradas apenas publicações dos últimos 10 anos, porém, por conta da escassez de dados, os últimos 20 anos foram considerados. A partir dessas informações, foram levantadas questões que conduziram esta pesquisa:

- Quais são os principais mecanismos de resistência apresentados por Acinetobacter baumannii?

- Quais as principais reações imunológicas associadas?

Para a pesquisa, foram utilizadas as seguintes palavras-chave: Acinetobacter baumannii, Infecção Nosocomial e Patógeno Oportunista. A leitura inicial dos resumos foi realizada e a separação foi feita de acordo com o tema abordado. Após leitura integral, os artigos condizentes com o assunto foram selecionados para embasar este estudo qualitativo de acordo com as normas de Pereira (2018).

\section{Resultados e Discussão}

De acordo com Cisneros e colaboradores (2008), a colonização da epiderme pode ser considerado o mais importante fator de risco para a disseminação da bactéria para outros pacientes inserido na mesma unidade de saúde e que necessitem de terapia respiratória com uso de equipamentos de ventilação, por conta da presença de biofilmes. Desse modo, pode ocorrer, ainda, transmissão direta pelas mãos dos profissionais que atuam nestes ambientes, por fômites, e diretamente de paciente para paciente (Munoz-Price \& Weistein, 2008).

Sendo que muitas Unidades de Terapia Intensiva (UTIs) brasileiras convivem atualmente com cepas de Acinetobacter baumannii resistentes a quase todos os antimicrobianos disponíveis, causando muitas Infecções Relacionadas à Assistência à Saúde (IRAS), essa problemática representa um alto custo financeiro no tratamento dos pacientes infectados e elevadas taxas de morte quando os antibióticos mais utilizados se tornam obsoletos (Ministério da Saúde, 2013).

Apesar de a resistência bacteriana ser, por si, um tema importante e atual, aparecendo como pauta em diversos meios de divulgação científica, existe uma dificuldade no que diz respeito à informação nas regiões norte e nordeste do Brasil: a escassez de trabalhos abordando este tema, com a finalidade de contribuir com dados epidemiológicos foi observada durante a busca de artigos confiáveis para a elaboração desta revisão de literatura.

São definidas como multirresistentes as cepas de A. baumannii que produzem carbapenases e apresentam resistência contra pelo menos três classes de antimicrobianos. São invulneráveis as Penicilinas de modo geral, cefalosporinas, aminoglicosídeos e fluoroquinolonas, mas susceptíveis aos antibióticos carbapenêmicos, amicacina, sulbactam e minociclina. 
As cepas pan-resistentes conseguem se opor mesmo frente a antibacterianos de amplo espectro, como polimixina e tigeciclina (Queenan, et al., 2014).

Um dos mais importantes mecanismos de resistência expressos por cepas de A. baumannii aos carbapenêmicos (doripenem, ertapenem, meropenem e principalmente imipenem), é a produção de carbapenemases, que hidrolisam também outros betalactâmicos, como cefalosporinas, penicilinas e monobactâmicos. Essa eficiência hidrolítica associada à sua codificação por genes localizados em elementos genéticos móveis, como plasmídeos e transposons, corrobora com sua disseminação em todo o mundo, resultando em alarmantes dados epidemiológicos e causando um impacto significativo na saúde humana à nível mundial (Djahmi, et al., 2014).

Três grandes classes de carbapenemases são encontradas atualmente em cepas de A. baumannii no mundo inteiro: as metalobetalactamases, sendo os tipos IMP, VIM e NDM. As mais frequentemente detectadas nas cepas de A. baumannii são as OXA- carbapenemases, sendo mais comum encontrar no Brasil as OXA-23, OXA-51, OXA-58 e OXA-143. Outros fatores relevantes são a produção dos genes $\mathrm{IpxC}$ e IpxD, que conferem resistência à Polimixina $\mathrm{B}$, além dos genes pmrA e pmrB. É preconizado pelo Ministério da Saúde em instituições hospitalares a implantação coleta de culturas de vigilância nas UTIs, de acordo com o perfil epidemiológico da instituição, através do uso de swabs em mucosas nasais, virilha, região perianal e pele. É realizado ainda o antibiograma para detecção fenotípica de resistência. Para pacientes colonizados, são indicadas medidas de contenção, sendo este cuidado realizado na admissão dos mesmos (Ministério da Saúde, 2013).

A infecção por este microrganismo em pacientes hospitalizados é um indicador risco de doenças graves. Um estudo realizado na UTI de um hospital terciário na cidade de Porto Alegre de março de 2006 a dezembro de 2008, acompanhou 66 pacientes positivos para Acinetobacter baumannii resistente a carbapenêmicos (ABRC), apresentando uma mortalidade de aproximadamente $47 \%$. Esta alta mortalidade se deve ao fato de os pacientes submetidos ao tratamento em uma Unidade de Terapia Intensiva apresentarem fragilidade imunológica e pela realização de procedimentos invasivos necessários para a manutenção da vida (Prates, 2010).

O sucesso de A. baumannii como patógeno humano parece estar intimamente ligado à facilidade com a qual esta bactéria realiza Transferência Horizontal de Genes (THG), que está intimamente relacionado à disseminação de genes associados a fatores de resistência. De acordo com vários estudos, este microrganismo é dotado de capacidade de se adaptar a diferentes habitats. A introdução de antibioticoterapia na década de 1950, levou à evolução e disseminação de alguns clones altamente homogêneos especificamente adaptado e resistentes ao ambiente hospitalar (Rangel, 2017).

Este microrganismo faz interessantes relações com o corpo humano, pois além de causar apoptose e dano tecidual pela produção de enzimas (Citocromo C), pode interferir na absorção de nutrientes, como o Ferro, por utiliza-lo em sua nutrição, com a finalidade de adentrar e se multiplicar nas células do hospedeiro. A captação do ferro ocorre pela excreção de receptores específicos em sua membrana, especialmente em tempos de escassez. A proteína OmpA pode causar apoptose nas células pois libera Citocromo C através de uma disfunção mitocondrial, resultando em dano tecidual (Goel \& Kapil, 2001).

\section{Conclusão}

O paciente colonizado por A. baumannii se torna um agente de transmissão indireta de patógeno ao ambiente hospitalar bem como a outros pacientes e aos profissionais da área da saúde, sendo que os clones circulantes são altamente resistentes a antimicrobianos (Scarcella, et al., 2017). Portanto estudar os modos de transmissão, assim como seu perfil de resistência, é de extrema relevância clínica e epidemiológica, uma vez que tal pesquisa poderá contribuir com ações mais assertivas no combate e prevenção de infecções causadas por este patógeno nas Unidades de Terapia Intensiva.

Diante do exposto, pode-se observar que algumas análises sobre a bactéria Acinetobacter baumannii merecem destaque, principalmente no que diz respeito a sua elevada capacidade de adaptação e de formar biofilmes em diversos 
ambientes. Quando formados em equipamentos hospitalares, que apresentam importância diferenciada, podem infectar e provocar o óbito de pacientes que apresentam insuficiência imunológica. Esta bactéria se comporta admitindo uma forma resistente que se propaga com facilidade, e tal invulnerabilidade a antimicrobianos mostra a importância de produzir conteúdo voltado ao tratamento e desenvolvimento de fármacos que possam agir de forma eficaz sobre esse agente e que, divergindo das medidas terapêuticas já existentes, obtenham efeitos colaterais menos agressivos e maior efetividade.

Sendo assim, vê-se que há a necessidade do desenvolvimento de novos estudos com abordagem para A. baumanii para que assim possamos ter melhor compreensão da sua dinâmica de transmissão, uma vez que esse patógeno apresenta a capacidade de gerar surtos, principalmente em ambientes hospitalares. Esse dinamismo merece ser investigado para que assim seja traçado um melhor perfil epidemiológico, mais próximo da realidade comportamental do A. baumanii, que, por consequência, irá contribuir com o manejo clínico de pacientes, pois uma vez conhecido o padrão de resistência, o tratamento poderá seguir uma linha maior de eficiência.

\section{Referências}

Ciello, G. \& Araújo, M. C. (2016). Perfil Epidemiológico do Acinetobacter baumannii Resistente a Carbapenens num Hospital do Interior Mineiro. 4(3), 20107. file:///C:/Users/WIN10/Downloads/1772-8859-4-PB.pdf.

Cisneros, J. M., Reyes, M. J., Pachón, J., Becerril, B., Caballero, F. J. \& Garcia-Garmendia, J. L. (1996). Bacteremia due to Acinetobacter baumannii: epidemiology, clinical findings and prognostic features. Clinical Infectious Diseases, 1026-32.

Dessel, H. V., Dijkshoom, L., Van der reijden, T., Bakker, N., Paauwa vanden broek, P., Verhoef, J. \& Brisse, S. (2004). Identification of a new geographically widespread multiresistant Acinetobacter baumannii clone from European hospitals. Research in Microbiology, 105-12.

Djahmi, Nassima, et al. (2014). "Epidemiology of carbapenemase-producing Enterobacteriaceae and Acinetobacter baumannii in Mediterranean countries." BioMed research international.

European Center for Disease Prevention and Control. (2008). https://www.ecdc.europa.eu/en/antimicrobial-resistance/facts/factsheets/experts.

Fournier, P. E. \& Richet, H. (2006). The epidemiology and control of Acinetobacter baumannii in health care facilities. Clin. Infect. Dis., $692-99$.

Goel, V. K. \& Kapil, A. (2001). Monoclonal Antibodies Against the Iron Regulated Outer Membrane Proteins of Acinetobacter baumannii are bactericidal. BMC Microbiology.

Merkier, A. K., \& Centrón, D. (2006). blaOXA-51-type $\beta$-lactamase genes are ubiquitous and vary within a strain in Acinetobacter baumannii. International journal of antimicrobial agents, 28(2), 110-113.

Ministério Da Saúde. (2013). Comissão Nacional de Prevenção e Controle de Infecções Relacionadas à Assistência à Saúde.

Munoz-price, L. S. \& Weistein, R. A. (2008). Acinetobacter Infection. New England Journal of Medicine, 358(12), $1271-81$.

Oplustil, C. P. (2004). Procedimentos básicos em microbiologia clínica. Sarvier, (2a ed.), 340.

Pereira, A. S. (2018) Metodologia da Pesquisa Científica, 67.

Perez, F., Hujer, A. M., Hujer, K. M., Decker, B. K., Rather, P. N. \& Bonomo, R. A. (2007). Global challenge of multidrug-resistant Acinetobacter baumannii. Antimicrobial Agents Chemother. Sarvier, 51(10), 3471-3484.

Prates, C. G. (2010). Mortalidade em uma unidade de terapia intensiva durante um surto de Acinetobacter baumannii resistente aos carbapenêmicos.

Prefeitura Minicipal De Porto Alegre. Manual de Orientação Para Controle da Disseminação de Acinetobacter, 06, 2007.

Rangel, L. T. L. D. O papel de transferência horizontal de genes na história evolutiva de duas classes de genes em bactérias (Doctoral dissertation, Universidade de São Paulo).

Queenan, K., Hasler, B. J. \& Rushton, A. (2014). One Health approach to antimicrobial resistance surveillance: is there a business case for it? International Journal of Antimicrobial Agents, Volume 48(4).

Scarcella, A. C., Scarcella, A. S. \& Beretta, A. L. R. (2017). Infecção relacionada à assistência à saúde associada a Acinetobacter baumannii: revisão de literatura - RBAC. 49(1), 18- 21.

Wroblewska, M. M., Dijkshoorn, L., Marchel, H., Van den Barselaar, M., Swoboda-Kopec, E., Van Den Broek, P. J., \& Luczak, M. (2004). Outbreak of nosocomial meningitis caused by Acinetobacter baumannii in neurosurgical patients. Journal of Hospital Infection, $57(4), 300-307$. 